\title{
Microbial Resistance in Urinary Tract Infections
}

\author{
Jahanzeb Malik $^{1}$, Nismat Javed ${ }^{2,1}$, Farhan Malik ${ }^{3}$, Uzma Ishaq ${ }^{4}$, Zubair Ahmed ${ }^{5}$ \\ 1. Cardiology, Rawalpindi Institute of Cardiology, Rawalpindi, PAK 2. Internal Medicine, Shifa College of \\ Medicine - Shifa Tameer-E-Millat University, Islamabad, PAK 3. Internal Medicine, Blackpool Teaching \\ Hospitals, NHS Foundation Trust, Blackpool, England, GBR 4. Hematology and Medical Oncology, Fauji \\ Foundation Hospital, Rawalpindi, PAK 5. Internal Medicine, Benazir Bhutto Hospital Rawalpindi, \\ Rawalpindi, PAK
}

Corresponding author: Nismat Javed, nismatjaved@gmail.com

\section{Abstract \\ Objective}

To determine the pattern of microbes responsible for urinary tract infections and their susceptibility to antimicrobial agents.

\section{Methods}

This was a prospective, observational study conducted at Benazir Bhutto Hospital, Rawalpindi, Pakistan. The urine samples of 440 patients were collected and sent for culture and sensitivity analysis. The results were recorded on a proforma. The data were analyzed using IBM Statistical Package for Social Sciences (SPSS) version 22 (IBM Corp., Armonk, NY). Descriptive statistics were used to describe the data. Chi-square test was applied to determine the significance of the difference between gender and microorganisms as well as microorganism and antimicrobial sensitivity. P-value of less than 0.05 was considered significant.

\section{Results}

Out of 440 urine samples, 144 culture-positive samples had been obtained from male participants and 296 culture-positive samples had been obtained from female participants. The most common organism on analysis was Escherichia coli. There were more rates of resistance in males. The organisms were most susceptible to fosfomycin and imipenem $(p=0.01)$. The organisms were resistant to ceftazidime $(p=0.01)$.

\section{Conclusion}

Received 05/01/2020

Review began 05/07/2020 Review ended 05/07/2020 Published 05/14/2020

\section{(c) Copyright 2020}

Malik et al. This is an open access article distributed under the terms of the Creative Commons Attribution License CC-BY 4.0., which permits unrestricted use, distribution, and reproduction in any medium, provided the original author and source are credited.
In Pakistan, most patients with resistance present with mild symptoms instead of severe clinical manifestations. Therefore, there is a need to reduce the over-prescription of antibiotics for urinary tract infections, especially in cases when other non-antimicrobial agents can be used.

Categories: Internal Medicine, Urology, Infectious Disease

Keywords: uti, pakistan, microbes, resistance

\section{Introduction}

Urinary tract infection is one of the common diseases present in the general population and 
affects a wide range of age groups [1,2]. The infections can involve both the upper and lower urinary tract, and therefore, patients present with a variety of symptoms. Cystitis, a lower urinary tract infection, manifests as dysuria, urgency, frequency, and sometimes, suprapubic tenderness. However, similar features are seen in upper urinary tract infections. Therefore, it becomes difficult to distinguish between the two unless there are other symptoms, such as a history of recurrence, anatomical malformations, fever, and systemic signs. In these few cases, the urinary tract infections are either complicated or uncomplicated [3]. There are many management options for urinary tract infections. In cases of acute and uncomplicated cystitis, fosfomycin-trometamol, nitrofurantoin, nitroxoline, pivmecillinam, and trimethoprim are firstline agents [4]. In cases of acute uncomplicated pyelonephritis, the drug of choice is fluoroquinolone [5]. Parenteral agents are indicated in cases of complicated urinary tract infections [6]. The decision regarding the drug of choice as empirical treatment becomes difficult in the current era of antimicrobial resistance $[7,8]$.

The objective of the study is to determine the pattern of bacterial agents responsible for urinary tract infections in a tertiary care hospital and to assess their antibiotic susceptibility and resistance pattern.

\section{Materials And Methods}

This is a prospective cross-sectional study that was conducted at Benazir Bhutto Hospital, Rawalpindi over a period of eight months. The sample size was calculated to be 440 urine samples using Open Source Epidemiologic Statistics for Public Health with a confidence interval of 95\%, a design effect of 1 for a random sample, and an anticipated frequency of $50 \%$ [9]. The expected population was estimated using the average monthly outpatient workload. The sampling technique was non-probability consecutive sampling. Informed consent was sought from all the participants. The study setting was the outpatient department of the hospital. The inclusion criterion was patients presenting with symptoms of urinary tract infection. The exclusion criteria of the study were patients who had refused to participate, patients who had been admitted to the hospital for management, and patients who had presented with a prediagnosed condition of the urinary tract. The urine samples of these patients were then taken to confirm the diagnosis. The culture and sensitivity results for the specimens were recorded on a proforma. The data were analyzed using IBM Statistical Package for Social Sciences (SPSS) version 22 (IBM Corp., Armonk, NY). Descriptive statistics were used to describe the data. Chi-square test was applied to determine the significance of the difference between gender and microorganisms as well as microorganism and antimicrobial sensitivity. Pvalue of less than 0.05 was considered significant.

\section{Results}

There were a total of 440 samples collected from patients. Out of 440 samples, 144 culturepositive samples had been obtained from male patients and 296 culture-positive samples had been obtained from female patients. The most common organism obtained from the urine culture was Escherichia coli (E. coli) (75\%). The organisms are further detailed in Table 1. 


\section{Cureus}

\begin{tabular}{|c|c|c|}
\hline \multirow{2}{*}{ Microorganism } & \multicolumn{2}{|l|}{ Gender } \\
\hline & Frequency of males (\%) & Frequency of females (\%) \\
\hline Escherichia coli & $107(24)$ & $223(51)$ \\
\hline Klebsiella & $13(3)$ & $32(7)$ \\
\hline Enterococcus spp. & $8(2)$ & $16(4)$ \\
\hline Staphylococcus aureus & $5(1)$ & $12(3)$ \\
\hline Pseudomonas aeruginosa & $7(2)$ & $5(1)$ \\
\hline Acinetobacter & $2(0)$ & $4(1)$ \\
\hline Enterobacter & $1(0)$ & $4(1)$ \\
\hline Proteus mirabilis & $1(0)$ & $0(0)$ \\
\hline
\end{tabular}

\section{TABLE 1: Microorganism yield in both genders}

The difference in the spectrum of microorganisms between the genders was not significant. The sensitivity of organisms to various antimicrobial agents was also noted and shown in Table 2. Chi-square test was applied to determine if the difference was significant. P-value of less than 0.05 was considered significant. 


\section{Cureus}

\begin{tabular}{|c|c|c|c|}
\hline Antimicrobial agent & Frequency of sensitive cultures (\%) & Frequency of resistant cultures (\%) & p-value \\
\hline Amoxicillin and clavulanic acid & $120(27)$ & $320(73)$ & 0.11 \\
\hline Aztreonam & $184(42)$ & $256(58)$ & 0.38 \\
\hline Amikacin & 435 (99) & $5(1)$ & 0.99 \\
\hline Gentamicin & $230(52)$ & $210(48)$ & 0.12 \\
\hline Cephalexin & $275(63)$ & 165 (37) & 0.22 \\
\hline Clarithromycin & $153(35)$ & $287(65)$ & 0.61 \\
\hline Ceftazidime & $93(21)$ & 347 (79) & 0.01 \\
\hline Ceftriaxone & $121(28)$ & $319(72)$ & 0.85 \\
\hline Ciprofloxacin & $153(35)$ & 287 (65) & 0.21 \\
\hline Nitrofurantoin & $406(92)$ & $34(8)$ & 0.75 \\
\hline Fosfomycin & $426(97)$ & $14(3)$ & 0.01 \\
\hline Imipenem & $376(85)$ & $64(15)$ & 0.01 \\
\hline Meropenem & $400(91)$ & $40(9)$ & 0.07 \\
\hline
\end{tabular}

\section{TABLE 2: Sensitivity of organisms to various antimicrobial agents}

$p<0.05$ was significant

The urine cultures from female patients had a wide spectrum of sensitivity (38.86\%) as opposed to samples that had been obtained from male patients (20.91\%). Escherichia coli was found to be sensitive to piperacillin and tazobactam (75.27\%), cefoperazone and sulbactam $(72.50 \%)$, vancomycin (4.54\%), azithromycin (0.22\%), teicoplanin $(0.22 \%)$, and tigecycline $(1.36 \%)$.

\section{Discussion}

The results of the study showed a 70:30 ratio between female and male participants in terms of culture-positive urine samples. This finding confirms the fact that females are at a higher risk of developing urinary tract infections $[10,11]$. This can be attributed to the fact that females have a short urethra [12]. Changes in estrogen levels are also responsible. Lower levels of estrogen cause the normal vaginal flora made up of Lactobacillus to decrease [13].

The most common organism was E. coli. Uropathogenic species of the organism possess various characteristics that provide them with an advantage over the normal immunity mechanisms. Fimbriae and pili increase the attachment of $E$. coli to host cells, whereas biofilm increases the adherence to the uroepithelium [14]. The presence of the biofilms also provides resistance against normal antimicrobial agents, which explains the high proportion of resistant cultures in our study.

The results showed that the majority of the cultures were sensitive to nitrofurantoin $(p=0.75)$ 
and fosfomycin $(p=0.01)$. The patients, therefore, might have presented with uncomplicated urinary tract infections and mild symptoms. This finding also implies that the urinary tract infection was caused by extended-spectrum $\beta$-lactamase $E$. coli. Nitrofurantoin and fosfomycin are recommended for first-line therapy in such subcategories because of the sensitivity of $E$. coli to both these agents [15].

This is a serious issue because extended-spectrum $\beta$-lactamase $E$. coli has a high sensitivity to ceftazidime and meropenem [16]. However, in our study, the majority of the cultures were found to be resistant to the agents. Furthermore, the sensitivity of the organism was high in the case of imipenem. This was a peculiar finding because there was a high rate of resistance for meropenem (91\%). Both these agents are considered highly efficacious for treating urinary tract infections but imipenem is emerging as a single agent therapy because of its pharmacodynamics characteristics [17].

A peculiar finding was the presence of significant resistance in cultures obtained from male participants, despite the lower yield of $E$. coli when compared to females. This could be related to the ratio of participants. This could also be related to the aging process in males. With increasing age, men tend to have voiding difficulties. These difficulties can generate turbulent retrograde urine flow leading to ascending infections. The prostate might be ultimately involved and it becomes a site for multiplication of resistant bacteria [18]. The extendedspectrum of resistance did not explain the sensitivity of $E$. coli to lactamase inhibitor combinations in drug therapy as well as macrolides and other agents because these species are more than likely to be resistant to the aforementioned drugs.

There are a few limitations to the study. The study was done at one centre. The study did not aim at dividing patients into appropriate age groups for risk stratification. The symptoms of the participants were not explored intensively. Antimicrobial agents within the same subgroup had not been compared.

\section{Conclusions}

Increasing antimicrobial resistance is a big concern for healthcare facilities worldwide. In Pakistan, the problem raises more concern, because most patients with resistance present with mild symptoms instead of severe clinical manifestations. There is a need to address the overprescription of antimicrobial agents to combat this problem.

\section{Additional Information}

\section{Disclosures}

Human subjects: Consent was obtained by all participants in this study. Rawalpindi Institute of Cardiology IRB \& EC issued approval 930-205-2016. Animal subjects: All authors have confirmed that this study did not involve animal subjects or tissue. Conflicts of interest: In compliance with the ICMJE uniform disclosure form, all authors declare the following:

Payment/services info: All authors have declared that no financial support was received from any organization for the submitted work. Financial relationships: All authors have declared that they have no financial relationships at present or within the previous three years with any organizations that might have an interest in the submitted work. Other relationships: All authors have declared that there are no other relationships or activities that could appear to have influenced the submitted work.

\section{References}

1. Pouladfar G, Basiratnia M, Anvarinejad M, Abbasi P, Amirmoezi F, Zare S: The antibiotic susceptibility patterns of uropathogens among children with urinary tract infection in Shiraz. 
Medicine (Baltimore). 2017, 96:e7834. 10.1097/MD.0000000000007834

2. Marsh KJ, Mundy L, Holter JJ, Johnson JR: Analysis of urine-specific antibiograms from veterans to guide empiric therapy for suspected urinary tract infection. Diagn Microbiol Infect Dis. 2019, 95:114874. 10.1016/j.diagmicrobio.2019.114874

3. Geerlings SE: Clinical presentations and epidemiology of urinary tract infections . Microbiol Spectr. 2016, 4:10.1128/microbiolspec.UTI-0002-2012

4. Kranz J, Schmidt S, Lebert C, et al.: The 2017 update of the German clinical guideline on epidemiology, diagnostics, therapy, prevention, and management of uncomplicated urinary tract infections in adult patients, part II: therapy and prevention. Urol Int. 2018, 100:271-278. 10.1159/000487645

5. Pietrucha-Dilanchian P, Hooton TM: Diagnosis, treatment, and prevention of urinary tract infection. Microbiol Spectr. 2016, 4:10.1128/microbiolspec.UTI-0021-2015

6. Desai S, Aronson PL, Shabanova V, et al.: Parenteral antibiotic therapy duration in young infants with bacteremic urinary tract infections. Pediatrics. 2019, 144:e20183844.

10.1542/peds.2018-3844

7. Wawrysiuk S, Naber K, Rechberger T, Miotla P: Prevention and treatment of uncomplicated lower urinary tract infections in the era of increasing antimicrobial resistance-non-antibiotic approaches: a systemic review. Arch Gynecol Obstet. 2019, 300:821-828. 10.1007/s00404-01905256-z

8. Wang A, Nizran P, Malone MA, Riley T: Urinary tract infections. Prim Care. 2013, 40:687-706. 10.1016/j.pop.2013.06.005

9. Open Source Epidemiologic Statistics for Public Health . (2013). Accessed: April 16, 2020: http://www.openepi.com/Menu/OE_Menu.htm.

10. Santoni N, Ng A, Skews R, Aboumarzouk OM: Recurrent urinary tract infections in women: what is the evidence for investigating with flexible cystoscopy, imaging and urodynamics?. Urol Int. 2018, 101:373-381. 10.1159/000490918

11. Stapleton AE: Urinary tract infection pathogenesis: host factors. Infect Dis Clin North Am. 2014, 28:149-159. 10.1016/j.idc.2013.10.006

12. Valiquette L: Urinary tract infections in women. Can J Urol. 2001, 8:6-12.

13. Stapleton AE: The vaginal microbiota and urinary tract infection . Microbiol Spectr. 2016, 4:10.1128/microbiolspec.UTI-0025-2016

14. Lagha R, Abdallah FB, Al-Sarhan BO, Al-Sodany Y: Antibacterial and biofilm inhibitory activity of medicinal plant essential oils against Escherichia coli isolated from UTI patients. Molecules. 2019, 24:E1161. 10.3390/molecules24061161

15. Meier S, Weber R, Zbinden R, Ruef C, Hasse B: Extended-spectrum $\beta$-lactamase-producing gram-negative pathogens in community-acquired urinary tract infections: an increasing challenge for antimicrobial therapy. Infection. 2011, 39:333-340. 10.1007/s15010-011-0132-6

16. Sternbach N, Weissman YL, Avni T, Yahav D: Efficacy and safety of ceftazidime/avibactam: a systematic review and meta-analysis. J Antimicrob Chemother. 2018, 73:2021-2029. 10.1093/jac/dky124

17. Zhanel GG, Lawrence CK, Adam H, et al.: Imipenem-relebactam and meropenemvaborbactam: two novel carbapenem- $\beta$-lactamase inhibitor combinations. Drugs. 2018, 78:65-98. 10.1007/s40265-017-0851-9

18. Smithson A, Ramos J, Niño E, Culla A, Pertierra U, Friscia M, Bastida MT: Characteristics of febrile urinary tract infections in older male adults. BMC Geriatr. 2019, 19:334.

10.1186/s12877-019-1360-3 